\title{
Towards Real-time Classification of Astronomical Transients
}

\author{
A. Mahabal*, S. G. Djorgovski*, R. Williams*, A. Drake*, C. Donalek*, M. \\ Graham* $^{*}$ B. Moghaddam ${ }^{\dagger}$, M. Turmon ${ }^{\dagger}$, J. Jewell ${ }^{\dagger}$, A. Khosla* and B. \\ Hensley*
}

${ }^{*}$ California Institute of Technology, MC 105-24, 1200 E. California Bl., Pasadena, CA 91125, USA

$\dagger$ Jet Propulsion Laboratory, 4800 Oak Grove Dr., Pasadena, CA 91109, USA

\begin{abstract}
Exploration of time domain is now a vibrant area of research in astronomy, driven by the advent of digital synoptic sky surveys. While panoramic surveys can detect variable or transient events, typically some follow-up observations are needed; for short-lived phenomena, a rapid response is essential. Ability to automatically classify and prioritize transient events for follow-up studies becomes critical as the data rates increase. We have been developing such methods using the data streams from the Palomar-Quest survey, the Catalina Sky Survey and others, using the VOEventNet framework. The goal is to automatically classify transient events, using the new measurements, combined with archival data (previous and multi-wavelength measurements), and contextual information (e.g., Galactic or ecliptic latitude, presence of a possible host galaxy nearby, etc.); and to iterate them dynamically as the follow-up data come in (e.g., light curves or colors). We have been investigating Bayesian methodologies for classification, as well as discriminated follow-up to optimize the use of available resources, including Naive Bayesian approach, and the non-parametric Gaussian process regression. We will also be deploying variants of the traditional machine learning techniques such as Neural Nets and Support Vector Machines on datasets of reliably classified transients as they build up.
\end{abstract}

Keywords: Transients; Classification; Bayesian Techniques; Machine Learning

PACS: 95.75.-z,95.80.+p,95.85.-e,95.85.Kr,98.70.-f

\section{INTRODUCTION}

Time domain astronomy has rapidly emerged as one of the more exciting areas of research in astronomy. It touches on a number of important scientific directions, ranging from exploration of the Solar System to cosmology. Besides the objects that move (e.g., asteroids, TNOs, KBOs), the types of transients we are likely to encounter include SNe (cosmological standard candles, as well as endpoints of stellar evolution), GRB orphan afterglows (which constrain the beaming models), variable stars of all sorts (probes of stellar astrophysics and Galactic structure), AGN (as a method of finding QSOs and constraining their fueling mechanisms and lifetimes), etc. There are classes of variable events which are expected or suspected to occur, but for which there is only a limited evidence in hand, e.g., tidal disruption of stars by otherwise quiescent supermassive black holes [1], breakout shocks of Type II SNe [2, 3], or mega-flares on otherwise normal, main-sequence stars [4], etc.

We have been exploring varibles and transients from the Palomar-Quest Sky Survey [12] (http: / /www.palquest.org) and the Catalina Sky Survey (http:

CP1082, Classification and Discovery in Large Astronomical Surveys, edited by C. A. L. Bailer-Jones

(C) 2008 American Institute of Physics 978-0-7354-0613-1/08/\$23.00 
/ /www. lpl arizona. edu/CSS) in real-time and announcing those via VOEventNet (http: / / voeventnet . cal tech . edu). Besides the optical surveys (e.g. PTF, LSST, Pan-STARRS) there are many at other wavelengths which will find transients and whose science can be enhanced by real-time classification of these transients (e.g. Fermi, SKA, LOFAR, LISA to name a few).

From targeted observing of small samples of a particular type of variable objects or phenomena, the field has been moving towards a systematic exploration of larger areas with a better time sampling and understanding of finer details of these phenomena. Rapid follow-up is essential for proper understanding and scientific exploitation of the events varying on a short time scale, or unusual classes of objects. Many of these objects do not have counterparts in archival image surveys like DSS, DPOSS, 2MASS etc. making the discovery data points and any follow-up the sole data to go on.

An illustrative example was OT SNF143933+054631 discovered in the Point-andStare data from Palomar-Quest by the LBNL SNF using archival comparison images. The initial SNIFs spectrum was highly unusual for a SN, and defied classification. Using follow-up imaging with the Palomar 60-inch telescope and a spectrum at Keck we were finally able to understand the nature of this peculiar SN [5]. Another case was SN20061t, which turned out to be a rare $\mathrm{SN}$ of type $\mathrm{Ib}[6,7]$. We may have discovered a class of SN associated with faint dwarf galaxies in the process of looking for transients [8].

SN and GRB are of course not the only transients for which unusual classes are found. CSS080924:233423+391423 seemed to be a simple flaring object until follow-up imaging revealed that it had persisted 24 hours later [9] and Fig. 1). A Palomar 200-inch spectrum revealed numerous emission lines at zero redshift typical of Galactic dwarf novae. The atypically large variations at discovery remain unexplained [10]. Yet another example is CSS080928:160837+041626, a possible high amplitude ( $\sim 5$ mags), long period variable, but with colors unlike one [11].

The need for quick reporting and follow-up has resulted in (1) the emergence of computer networks and protocols for collecting and distributing streams of interesting events from large surveys - the VOEventNet system which serves events from a multitude of streams including Palomar-Quest survey and the Catalina Sky Survey is a pertinent example [12], and (2) a number of robotic telescopes which can turn to a target very quickly and provide crucial data for the classification of the events.

Here we describe the current status of the real-time event classification effort. The endeavour is clearly applicable to other synoptic sky surveys. As the event streams from synoptic sky surveys such as LSST and SKA increase, real-time classification will become even more crucial as there will not be enough facilities for follow-up observations making real-time classification a key enabler of future synoptic astronomy.

A key difficulty of real-time classification of transients is the general lack of available information initially available. A transient detected by an increase in brightness is often missing in archival sky surveys and may have just a couple of relatively closely spaced observations in a couple of epochs to go by. Machine Learning methodologies including Support Vector Machines (SVMs), Artificial Neural Networks (ANNs) can be used as also Bayesian classifiers including Naive Bayesian algorithms and Gaussian Process regression [13]. 


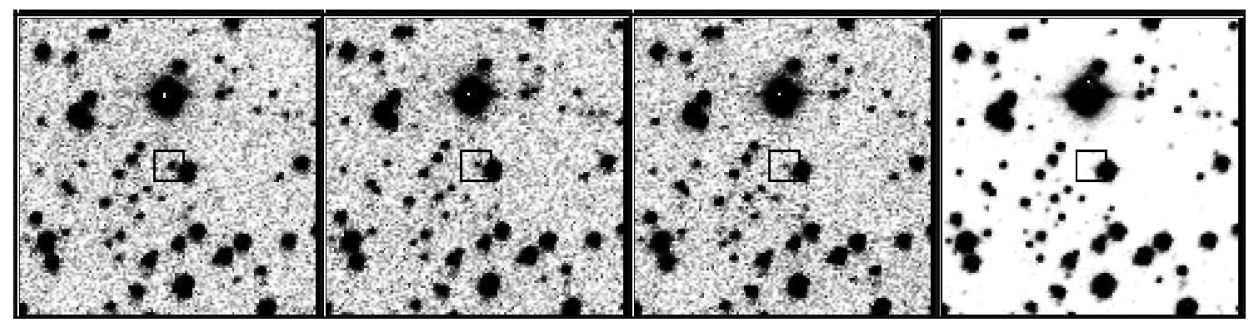

FIGURE 1. CSS080924:233423+391423 was classified as a transient based on its flaring within the CSS images taken minutes apart. Not found in any archival surveys except for the DSS N plate, it was still bright the next day. A spectrum later revealed it to be a dwarf nova, but its rapid variation remains unexplained. The three images on the left are individual CSS images taken on Sep 242008 UT, and the one on the right is a co-added baseline image, also from CSS.

\section{METHODOLOGY}

\section{Bayesian Event Classification}

Given a small number of observations, it is not generally possible to unambiguously classify transients. The best approach in such a case is to calculate the probabilities of the object belonging to different classes of transients and then using objective criteria to determine if the probability for the class of interest is high enough. There will also be cases when none of the known classes is a good fit. This type may perhaps turn out to be most interesting, with the transient being a possible example of a new type of astronomical object or phenomenon.

We have described such a probabilistic method utilising Naive Bayes method in [14]. Briefly, the method involves building priors of different types of objects with a large number of features. The object to be classified has a feature vector which is decomposed into several independent blocks based on which class is being considered. While this does not allow an exact membership to be determined, obtaining approximate probabilities while circumventing the curse of dimensionality as well as coping with several missing values makes this a powerful method. See also $[15,16]$.

\section{Machine Learning in Classification}

The machine learning, using ANNs/SVMs is more useful for dealing with variables where more prior data is available [14]. These too can deal with incomplete information, at least partially, by training several sets of quasi-independent classifiers and invoking the appropriate ones depending on what information is available (magnitudes, colors, shape parameters etc.).

Another way we have started to use these techniques is to classify (and eliminate) artifacts in real-time as data from telescopes are being processed. Many artifacts, based on some features present in their signature and the fact that they may not correspond to an object in the fiducial images, can get initially flagged as transients. This technique 
helps remove them. More details can be found in [17]. Similarly a very pertinent issue is star-galaxy classification from multi-epoch data. Due to varying conditions (seeing, airmass, extinction, filter) it is not possible to reproduce the same class in each individual epoch. Combining the disparate data can be ideally carried out using ANNs [17].

\section{Feedback Incorporation}

Feedback incorporation [14] is an important step since every bit of additional information can potentially vindicate or contradict the original classification (or at least revise probabilities). In addition, the priors should also be updated so that classification in future has access to the new information for the corresponding classes. This can be carried out using either Expectation-Maximization algorithm [18] or kernel density estimation [19]. The unknown parameters in these approaches can be determined using known physical parameters for given classes (e.g. SNs do not normally increase in brightness once they start fading, or RR-Lyrae do not have their own host galaxies etc.) or additional observations as they become available or labelling done by experts. This final bit plays an important role in semantically connected portfolios built for each transient. Such transient portfolios are structured yet flexible annotation mechanisms including images and spectra besides comments. The collection of portfolios can be indexed for ease of searching and execution of need-based services [20].

\section{Follow-up Prioritization Engine}

Given the paucity of follow-up resources in the era of very large volume event streams, matching transients to follow with such resources may well be the most crucial step in advancing our knowledge about rare classes of quickly fading objects. It will also be crucial in breaking the ambiguity between two possible classes. An informationtheoretic approach leading to the reduction of final entropy is the best choice $[21,14]$.

\section{Gaussian Process Regression}

Given a small number of observations in a single band at different epochs, Gaussian Process Regression (GP) can be an effctive in determining class membership. In this process priors are built from lightcurves of objects belonging to a class of objects. For instance, consider just two epochs of a newly discovered transient (Fig. 2). One can compare the two points against different parts of a lightcurve in order to determine if there is a possible fit and if so at what stage of evolution (e.g. periodicity). The lower panel shows such a comparison with log marginal likelihood on the Y-axis. The more observed points one has, the more stringent results one can obtain. Fig 3 shows different possibilities involving 3 points (i.e. $2 d t$ and corresponding $d m$ ). For a given class of objects one can visualize a surface made of a grid of $\mathrm{dt}$ and $\mathrm{dm}$ values which can 

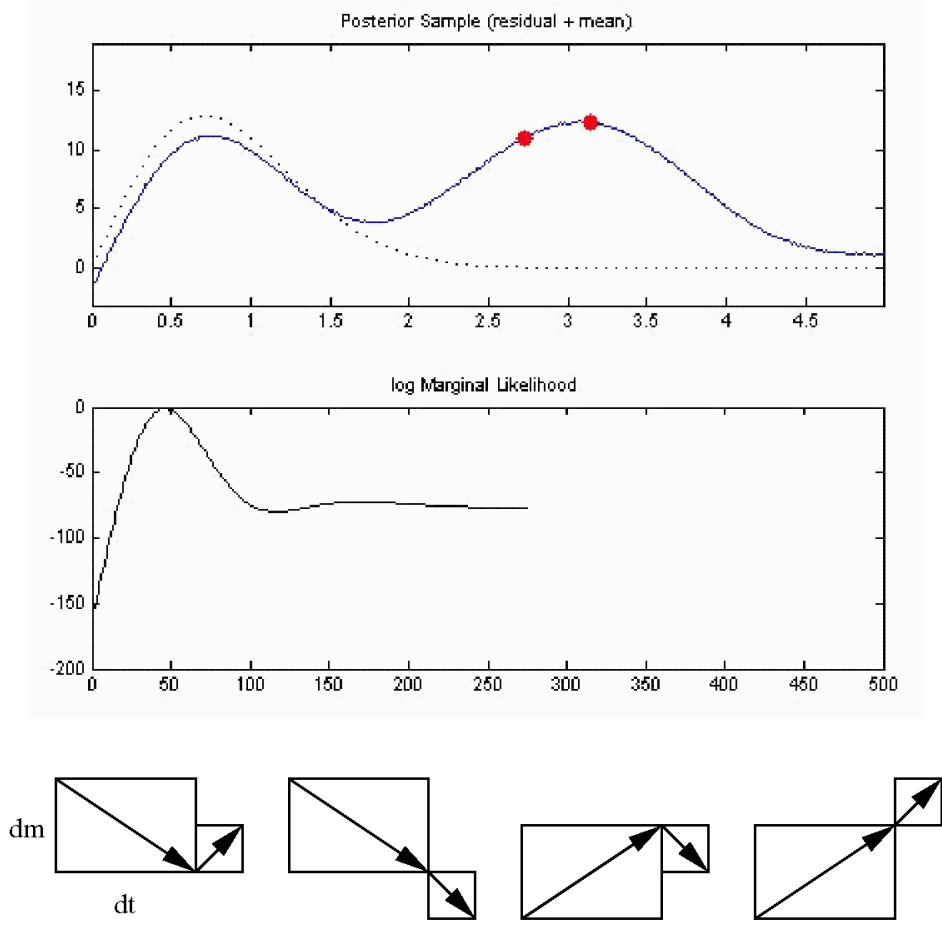

FIGURE 2. Gaussian Regression for a given $d m$ over the corresponding $d t$. The second panel shows log marginal likelihood of the pair corresponding to different parts of the given lightcurve. When more points are available for comparison, it is easier to eliminate a larger number of priviously competing hypotheses. The boxes below show the distinct possibilities when three observations are present. The lightcurve, within error-bars should accomodate the end-points of the arrows to be a valid choice.

then be used to determine class membership. However, owing to the variation in objects belonging to a class, such a surface is somewhat fuzzy.

We have been conducting preliminary investigations using SN and Mira light curves and will be extending it to other light curves. The results so far are encouraging.

\section{VOEventNet}

VOEventNet (http : / / voeventnet . cal tech . edu) federates streams of astronomical events such that humans as well as robotic telescopes can subscribe to the events. The events are available in real-time in a standard format. The different streams allow subscribers to choose events of their interest (e.g. SN, GRB, asteroids etc.). Google Sky serves VOEventNet events under their "Current Sky Events" with links to related technical and astronomical information. A color scheme allows more recent events to stand out. AAVSO streams are also expected to be available under VOEventNet soon. 


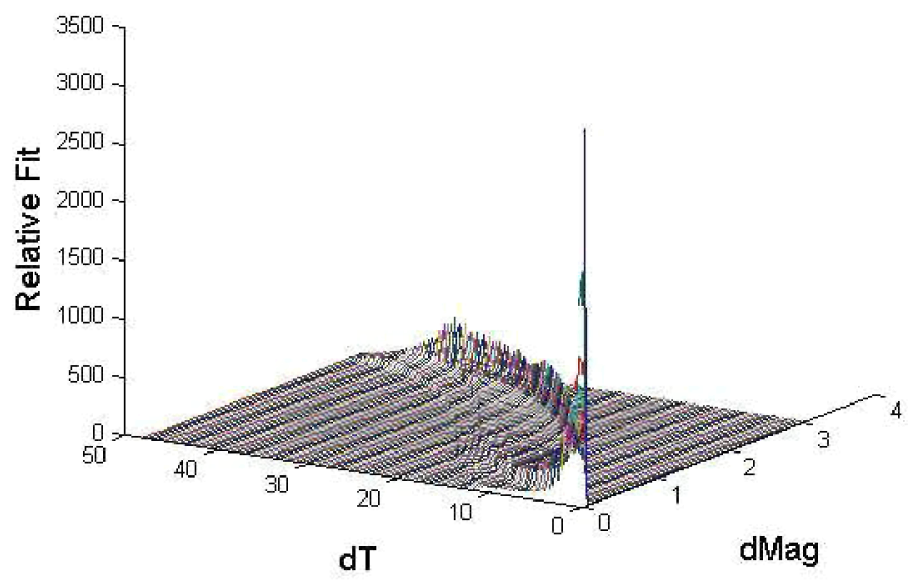

FIGURE 3. Given an object type one can build a surface indicating what likely $d m$ s at what $d t \mathrm{~s}$ one is likely to encounter. One can then compare any such pair against such a surface to see if the pair of points come from an object of that type. In practise we of course do not have such a crisp surface.

The reecntly concluded Palomar-Quest survey and the ongoing Catalina Sky Survey utilize VOEventNet to serve variables and transients in real-time. This allowed quick follow-up in many cases and has resulted in datasets which would otherwise be difficult to compile. For instance, during the first six months of its real-time observations, CSS found 350 transients [8]. 240 of these were SN or CV and the remaining included AGNs, high proper motion stars, highly variable stars as well as blazars and transients of an unknown nature.

We have been conducting follow-up of many of the interesting transients and variables at the palomar 60-inch telescope. These additional data aid in classification as well as in the enhancement of priors for the Bayesian and machine learning modules.

\section{SUMMARY}

Many of the components described above are under development while some of them exist and prototypes have been succesfully used to detect variables and transients in PQ and CSS. But with event streams poised to become larger and larger, the methodology and the architecture will have to be refined so that problem-specific, on-demand computing services can compute/recompute virtual data according to a particular algorithm as the need arises. This will have to be explicitly linked using transient portfolios to various products like "baseline sky" used in the detection and characterization of transient phenomena. Reaction and redirection of measurement processes are needed. A proper, 
scalable workflow incorporating these components will have to be realized to make full use of the forthcoming event streams.

We have presented the status of real-time classification of events and on-going developments including Bayesian networks and Machine Learning (ML) techniques. Feedback from follow-up observations is necessary to improve priors but will be increasingly scarce compared to the volume of event streams making it important to continuously update training data sets. The implementations of the classification methodology used in PQ/CSS along with VOEventNet framework should help improve scientific returns from future synoptic sky surveys.

\section{ACKNOWLEDGMENTS}

We are grateful to the staff of Palomar Observatory for their help, and to our collaborators in PQ and CSS survey teams. This work was supported in part by the NSF grants AST-0407448, AST-0326524, and CNS-0540369, and by the Ajax Foundation. A.K. and B.H. were supported in part by the Caltech SURF program.

\section{REFERENCES}

1. S. Gezari et al., 2006, ApJ, 653, L25

2. K. Schawinski et al., 2008, Science, 321, 223

3. A. Soderberg et al., 2008, Nature, 453, 469

4. S. G. Djorgovski et al. in Mining the Sky, edited by A.J. Banday et al., ESO Astrophysics Symposia, Berlin: Springer Verlag, 2000, 305.

5. A. Mahabal et al., 2006, Astronomer's Telegram 827

6. E. Pecontal, et al., 2006, Astronomer's Telegram 918

7. A. Soderberg et al., 2006, Central Bureau Electronic Telegrams 714

8. A. Drake et al., 2008, submitted to ApJ, arXiv:0809.1394

9. A. Mahabal et al., 2008, Astronomer's Telegram 1741

10. R. Quimby et al., 2008, Astronomer's Telegram 1750

11. A. Drake et al., 2008, Astronomer's Telegram 1768

12. S. G. Djorgovski et al., 2008, AN, 329, 263

13. C. E. Rausmussen \& C. K. I. Williams, in Gaussian Processes for Machine Learning, The MIT Press, 2002.

14. A. Mahabal et al., 2008, AN, 329, 288

15. D. J. Hand \& K. Yu, 2001, International Statistical Review, 69, 385

16. P. Domingos \& M. Pazzani, 1997, Machine Learning, 29, 103

17. C. Donalek et al., 2008, this volume

18. M. Turmon, J. M. Pap, \& S. Mukhtar, 2002, ApJ, 568, 396

19. B. W. Silverman, 1986, Density Estimation for Statistics and Data Analysis, Chapman and Hall pub1.

20. M. Graham et al., 2008, ADASS, in prep.

21. T. J. Loredo, \& D. F. Chernoff, 2003, Statistical Challenges in Modern Astronomy III (eds. E. D. Feigelson and G. J. Babu, Berlin: Springer Verlag), 57 